\title{
Feasibility and Acceptability of a Resistance Exercise Intervention: For Women Undergoing Chemotherapy for Breast Cancer
}

\author{
Constance Visovsky ${ }^{*}$, Janique Corripio \\ College of Nursing, University of South Florida, USA
}

Copyright (C) 2015 by authors, all rights reserved. Authors agree that this article remains permanently open access under the terms of the Creative Commons Attribution License 4.0 International License

\begin{abstract}
Resistance exercise can maintain muscle strength \& physical functioning in women undergoing chemotherapy for breast cancer. Few studies examined resistance exercise for reducing the neuromuscular side effects of chemotherapy. The purpose of this analysis was to examine the feasibility and acceptability of a resistance exercise intervention for women undergoing chemotherapy for the treatment of breast cancer. The data reported are from the final data collection interview of the intervention group $(\mathrm{n}=47)$ who participated in a randomized clinical trial testing a resistance exercise intervention for women with breast cancer. Data regarding feasibility and acceptability was collected using an investigator-developed questionnaire consisting of 6 Likert-scale items (range $=1-5$ ), and 4 additional open-ended questions concerning exercise benefits and barriers. Descriptive statistics were used to analyze the Likert-scale questions. A mean score of 3.0 or greater indicated that the intervention was acceptable. A content analysis was performed on the open-ended items. Five of six questions exceeded the 3.0 set point indicating women found the remaining aspects of the intervention feasible and acceptable. Challenges in exercise were symptoms, fatigue, and time constraints. The benefits from the exercise program consisted of increased strength and energy, emotional well-being, physical well-being. Study results suggest that resistance exercise intervention conducted during chemotherapy for breast cancer is feasible and acceptable. Social support for exercise was the most important factor in continuing an exercise program during chemotherapy. Study participants can be successfully recruited into a resistance exercise program during chemotherapy for breast cancer. However, stronger emphasis in symptom management during the early phases of the study is needed to prevent study withdrawal.
\end{abstract}

Keywords Breast Cancer, Resistance Exercise, Feasibility

\section{Introduction}

There is ample evidence to suggest that exercise can improve body composition, enhance muscular endurance, flexibility and quality of life (QOL) in women with breast cancer. A recent meta-analysis by Schmitz, et al (2005) ${ }^{[1]}$ examining the effect of physical activity in cancer survivors noted that inactivity both during and following cancer treatment can have ill effects on cardiorespiratory functioning, bone mineralization, muscle strength, glucose metabolism, insulin sensitivity, digestive and immune functioning. Although many studies of exercise for women with breast cancer have been conducted, these studies primarily enrolled women with early stage disease and used only aerobic exercise as the intervention. $[2,3,4,5,6,7,8,9,10,11,12,13,14]$ There have been several studies testing the effects of resistance exercise in women with breast cancer. These studies recruited women into resistance exercise interventions following the completion of cancer treatment, with the exception of long-term anti-estrogen therapy. ${ }^{[15,16,17]}$ The study outcomes examined were varied, and included anthropometric measures, physical activity, food and nutrient intake, fasting blood glucose, plasma insulin levels, insulin resistance, quality of life, depressive symptoms, dynamic balance, muscle strength, and bone mineral density. Engaging in resistance exercise did not appear to induce or worsen lymphedema in women who had undergone lymph node dissection. ${ }^{18}$ It is important to note that chemotherapy treatment protocols for breast cancer continue to evolve. Newer dose-dense chemotherapy regimens consisting of four cycles of doxorubicin (Adriamycin) and cyclophosphamide (Cytoxan) followed by paclitaxel (Taxol) administered every 2 weeks with concurrent growth factor support are becoming more common. This change in clinical practice has allowed breast cancer chemotherapy to be condensed over a shortened time period with improved outcomes. [19] While this chemotherapy dosing schedule has increased survival for women diagnosed with the disease, it has also induced 
significant neuromuscular toxicity.

Interventions aimed toward assisting women in the prevention or management of chemotherapy-induced neuromuscular toxicity are lacking. Few studies have used resistance exercise as a sole modality aimed at reducing the neuromuscular side effects of chemotherapy. Newer interventions require an assessment of their feasibility and acceptability to the populations for which they were developed. The lack of literature concerning the feasibility of resistance exercise underscores the need for assessment of such interventions. Thus, the purpose of this paper is to report the feasibility and acceptability of a resistance (strength training) exercise intervention for women undergoing chemotherapy regimens for the treatment of breast cancer.

The data reported here represent the final (12 week) data collected as part of a randomized clinical trial of a resistance exercise (strength training) intervention for women undergoing chemotherapy as part of their breast cancer treatment. Study participants who were randomized to receive the resistance exercise intervention completed an investigator-developed Participant Evaluation of Feasibility and Acceptability Questionnaire to assess feasibility and acceptability of the home-based strength training intervention.

\section{Conceptual Framework}

A physiological framework formed the basis for the study. The administration of chemotherapy exposes the individual to exogenous and endogenous cytokines that have the capability of inducing pathological changes in the muscle, resulting in increased fatigability and muscle weakness. In addition, physical fatigue as a common side-effect of chemotherapy, leads individuals to become inactive. Inactivity in turn induces deteriorations in muscle structure, function, and strength. ${ }^{[20]}$ Disuse atrophy then follows with the most profound changes occurring in the postural muscles of the leg and back. ${ }^{[21]}$ Metabolic changes in the muscles, and subsequent declines in muscle force generating capacity pre-dispose the individual to further muscle weakness, resulting in a decline in functional performance. ${ }^{[22]}$

\section{Methods}

\section{Design}

The data reported here are from the final (12 week) data collection interview of the intervention group who participated in a prospective, longitudinal randomized clinical trial testing a resistance exercise intervention for women with breast cancer receiving chemotherapy. Study participants were randomized to either the resistance exercise intervention or an attentional control group following baseline data collection.

The primary aim of the study was to determine the effect of a resistance exercise program on changes in muscle strength, fatigue, body composition and quality of life. Outcome variables were measured as follows: muscle strength was measured using a hand-held dynamometer; fatigue was measured using the Piper Fatigue Scale ${ }^{[24]}$; body composition was measured using bioelectrical impedance analysis (BIA) with a Tanita body fat monitor (Tanita, model TBF-52) to obtain fat mass and fat-free or lean body mass; quality of life was measured using the SF-36 Health Survey version 2.0. ${ }^{[25]}$ The secondary aim was to determine the feasibility and acceptability of a home-based resistance exercise program.

\section{Sample \& Setting}

The study sample consisted of 100 women with Stage I-IIIa breast cancer recruited from two Midwestern cities. The sample for this reported analysis consists of the women randomized to the resistance exercise intervention $(n=47)$. Table 1 lists the characteristics of the sample. Women were eligible for the study if they were going to receive chemotherapy as part of their breast cancer treatment plan; were age 21 or older; had no previous treatment with chemotherapy; no disease or prior illness associated muscle weakness (such as chronic fatigue syndrome, multiple sclerosis, spinal cord tumors or injuries, stroke, preexisting cardiopulmonary disease); no evidence of bone metastasis or osteoporosis; and no medications known to have deleterious effects on the muscles (i.e. statins). These criteria were selected to reduce the number of confounding variables.

\section{Data Collection \& Procedures}

Institutional Review Board approval was received from the two Midwestern cancer centers for the conduct of the study. Medical oncologists and their associated clinical nurses referred eligible women to the investigator for study participation. Following referral, each participant was provided with information about the study by a trained member of the research team. Written informed consent was obtained and a copy of the signed consent was given to each woman. Study participants randomized to receive the resistance exercise intervention were given wrist and ankle weights, a pictorial Exercise Guide with accompanying instructions on performing the various exercises, and an Exercise Diary in which to record the amount of weight used, the number sets and repetitions done, and the number of exercise sessions per week. Exercise Diaries were collected by the research staff at 4,8 and 12 weeks of the intervention. The intervention protocol consisted of exercises targeting both upper and lower extremities performed 3 times weekly The strength training exercises consisted of bicep curls, triceps extensions, front and lateral raises, shoulder press, calf raises, lunges, supine leg curls and supine leg extensions. Strength training exercises initially began with performing 1-2 sets of each exercise for 8 repetitions 1-2 times per week. At week 4, the number of sets was increased to 2-3 sets and 8-12 repetitions of each exercise per session for the remainder of the study period. 
112 Feasibility and Acceptability of a Resistance Exercise Intervention: For Women Undergoing Chemotherapy for Breast Cancer

Table 1. Sample Characteristics

\begin{tabular}{|c|c|c|}
\hline Characteristic & $\mathbf{n}$ & $\%$ \\
\hline \multicolumn{3}{|l|}{ Age (years) } \\
\hline \multicolumn{3}{|l|}{$X=51.15$} \\
\hline \multicolumn{3}{|l|}{ Range $=26-73$} \\
\hline \multicolumn{3}{|l|}{$\mathrm{SD}=10.11$} \\
\hline \multicolumn{3}{|l|}{ Ethnicity } \\
\hline Caucasian & 92 & 92 \\
\hline African American & 7 & 7 \\
\hline Other & 1 & 1 \\
\hline \multicolumn{3}{|l|}{ Marital Status } \\
\hline Married & 76 & 76.8 \\
\hline Separated & 2 & 2.0 \\
\hline Divorced & 11 & 11.1 \\
\hline Single & 6 & 6.1 \\
\hline Widowed & 2 & 2.0 \\
\hline Other & 2 & 2.0 \\
\hline \multicolumn{3}{|l|}{ Currently employed } \\
\hline Yes & 67 & 32.3 \\
\hline No & 32 & 67.7 \\
\hline \multicolumn{3}{|l|}{ Exercise } \\
\hline Yes & 43 & 43.4 \\
\hline No & 56 & 56.6 \\
\hline \multicolumn{3}{|l|}{ Exercise routine } \\
\hline Aerobic & 23 & 23.2 \\
\hline Weights/Resistance exercise & 2 & 2.0 \\
\hline Combination & 11 & 11.1 \\
\hline Yoga/Comparable activity & 5 & 5.1 \\
\hline None/No exercise & 49 & 49.5 \\
\hline \multicolumn{3}{|l|}{ Stage of breast cancer } \\
\hline Stage 1 & 232 & 24.7 \\
\hline Stage 2 & 53 & 57.0 \\
\hline Stage 3 & 15 & 16.1 \\
\hline Stage $3 \mathrm{a}$ & 2 & 2.2 \\
\hline \multicolumn{3}{|l|}{ Type of surgery } \\
\hline Lumpectomy & 50 & 54.9 \\
\hline Modified Radical Mastectomy & 15 & 16.5 \\
\hline Total Mastectomy & 17 & 18.7 \\
\hline No surgery/Biopsy only & 6 & 6.6 \\
\hline \multicolumn{3}{|l|}{ Lymph node dissection } \\
\hline Yes & 89 & 91.8 \\
\hline No & 8 & 8.2 \\
\hline \multicolumn{3}{|l|}{ Number of lymph nodes dissected } \\
\hline $1-7$ & 38 & 44.7 \\
\hline $8-14$ & 31 & 36.5 \\
\hline $15-21$ & 11 & 12.9 \\
\hline $22-28$ & 4 & 4.7 \\
\hline$>29$ & 1 & 1.2 \\
\hline \multicolumn{3}{|l|}{ Dose dense } \\
\hline Yes & 51 & 85.0 \\
\hline No & 9 & 15.0 \\
\hline
\end{tabular}

*Some data may be missing contributing to the percentages not equaling 100. 
Resistance exercises were taught by trained study personnel under the guidance of an exercise physiologist. A pictorial Exercise Guide was given to each intervention group study participant as a reference. Resistance exercise intervention participants were instructed to perform the various exercises three times weekly using an individualized, pre-determined amount of weight for the upper and lower extremities. Data concerning each woman's pattern of exercise and all study outcomes were measured prior to chemotherapy (baseline), and 4 weeks, 8 weeks and 12 weeks of chemotherapy treatment.

Data regarding the feasibility and acceptability of the resistance exercise program were collected at the end of the resistance exercise intervention (12 weeks) using an instrument developed specifically for this study by the principal investigator. The Participant Evaluation of Feasibility and Acceptability Questionnaire consists of 6 items arranged on a Likert scale ranging from 1- 5, and 4 additional open-ended questions designed to elicit information concerning the benefits and barriers to participating in the resistance exercise intervention while undergoing chemotherapy for breast cancer. The Feasibility and Acceptability Questionnaire consists of six questions, five questions related to the assigned exercises and one question related to exercise guidance received. The participants addressed the following questions: I found the exercises fun to do; I was able to do the exercises without difficulty; the exercises were worth my time to do; the exercises were easy to do; the directions for doing the exercises were clear; and I received the guidance I needed from the research nurses. The scale consisted of five choices: 1 (strongly disagree), 2 (disagree), 3 (agree), 4 (moderately agree), and 5 (strongly agree). A mean score of 3.0 or greater was set apriori as the study criterion to indicate that the intervention was acceptable.

Four additional open ended questions followed to assist in identifying what was most helpful in the continuation of exercise; the biggest challenges to exercise; benefits received from participating in the exercise program; and suggestions for other similar individuals. A content analysis was performed for the open-ended items. Feasibility and acceptability of the intervention was also measured by calculating the recruitment rate, adherence to the intervention protocol, and withdrawal rates among study participants.

\section{Data Analysis}

Data analysis consisted of descriptive statistics using SPSS (version 14.0). The total number of participants in the study consisted of 100 women with 47 women randomized to the intervention group. Thirty three women $(70.2 \%)$ actually completed the entire 12 week intervention. Twenty-eight women (84.8\%) completed the Participant Evaluation of Feasibility and Acceptability questionnaire at the end of the 12 week intervention.

Table 2. Feasibility and Acceptability Questionnaire

\begin{tabular}{|c|c|c|}
\hline Questions & Mean & $\begin{array}{c}\text { Standard } \\
\text { Deviation }\end{array}$ \\
\hline I found the exercises fun to do & 2.33 & 0.99 \\
\hline I was able to do the exercises without \\
difficulty & 3.26 & 1.19 \\
\hline The exercises were worth my time to do & 3.85 & 1.06 \\
\hline The exercises were easy to do & 3.52 & 1.05 \\
\hline $\begin{array}{c}\text { The directions for the exercises were } \\
\text { clear }\end{array}$ & 4.07 & 0.99 \\
\hline $\begin{array}{c}\text { I received the guidance I needed from the } \\
\text { research nurses }\end{array}$ & 4.33 & 0.92 \\
\hline
\end{tabular}

The first question of the Feasibility and Acceptability Questionnaire addressed enjoyment of resistance exercise had a mean score of $2.75(\mathrm{SD}=0.99)$, indicating a lack of acceptability for this item on the scale. The remaining five questions had a mean score greater than the 3.0 minimum criteria, indicating women found the remaining aspects of the intervention feasible and acceptable. The questionnaire also contained 4 open-ended questions used to inform the research team of helpful assistance, perceived benefits, challenges, and suggestions to improve the exercise program from the perspective of the participants. Not all of the 28 respondents provided answers to the open-ended items on the Feasibility and Acceptability Questionnaire. Figures 1-4 denote the response to the open-ended questions. A content analysis was performed on the four open-ended questions. The research team reviewed all the responses to the open-ended questions independently and coded responses into themes. A qualitative researcher reviewed the themes for agreement and consistency. 


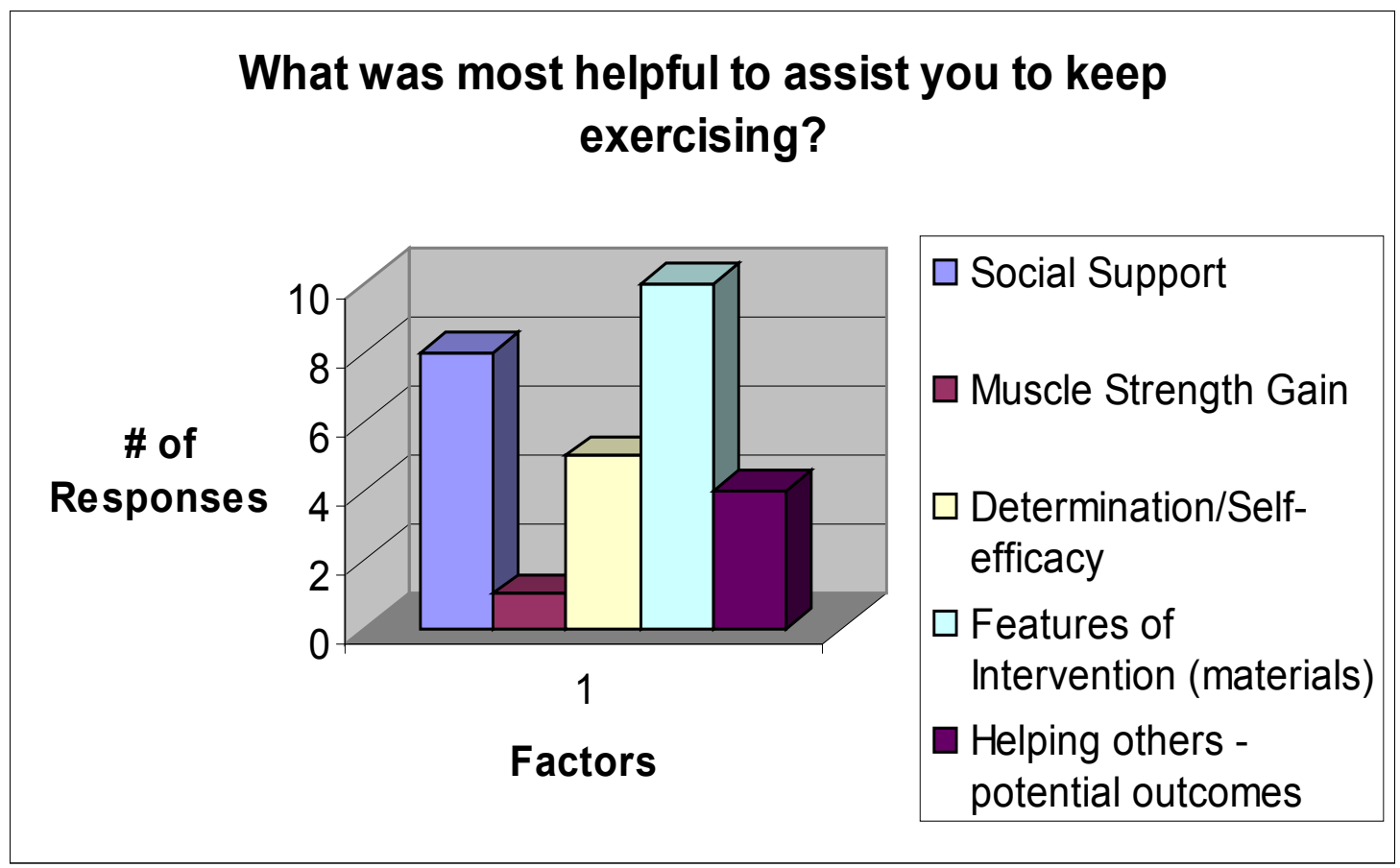

Figure 1. Feasibility Question 1

\section{What were the biggest challenges that kept you from doing the exercises?}

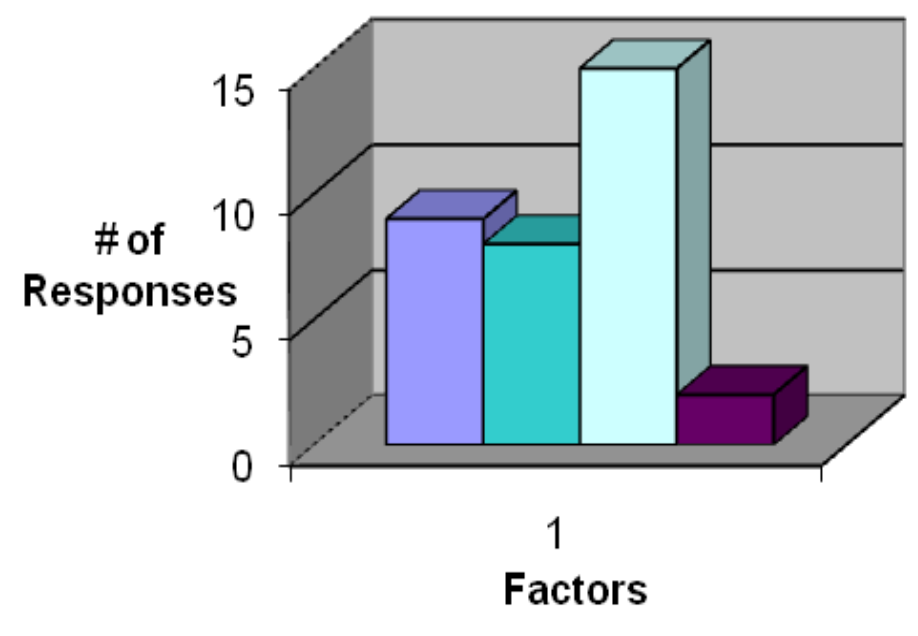
口Fatigue
口Time Constraints
口Other Symptoms (muscles aches)
CChallenges related to intervention

Figure 2. Feasibility Question 2 


\section{What benefits did you feel you received from your participation in the exercise program?}

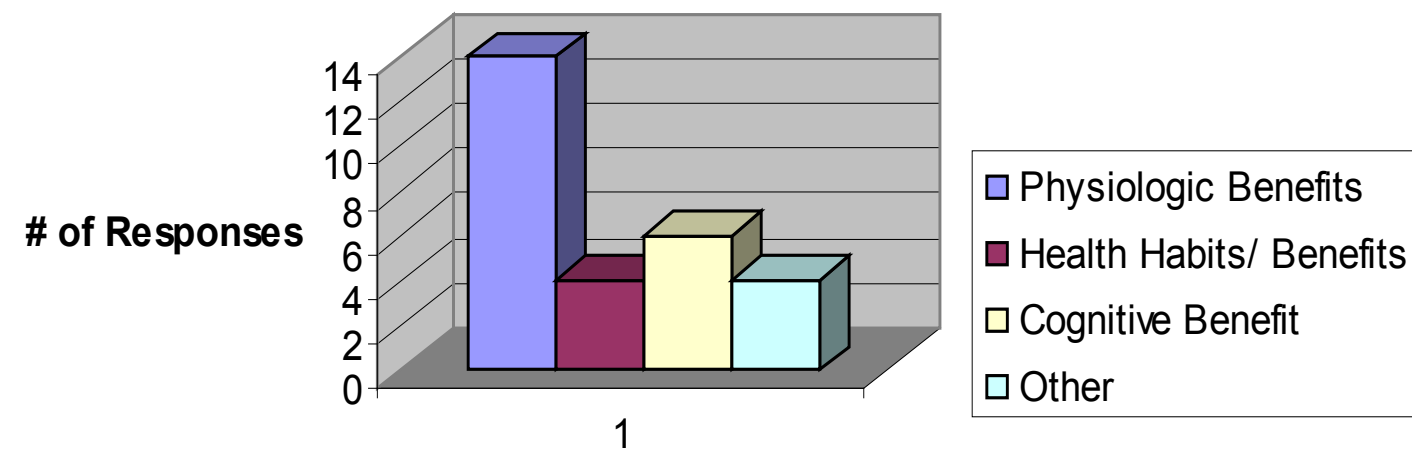

Factors

Figure 3. Feasibility Question 3

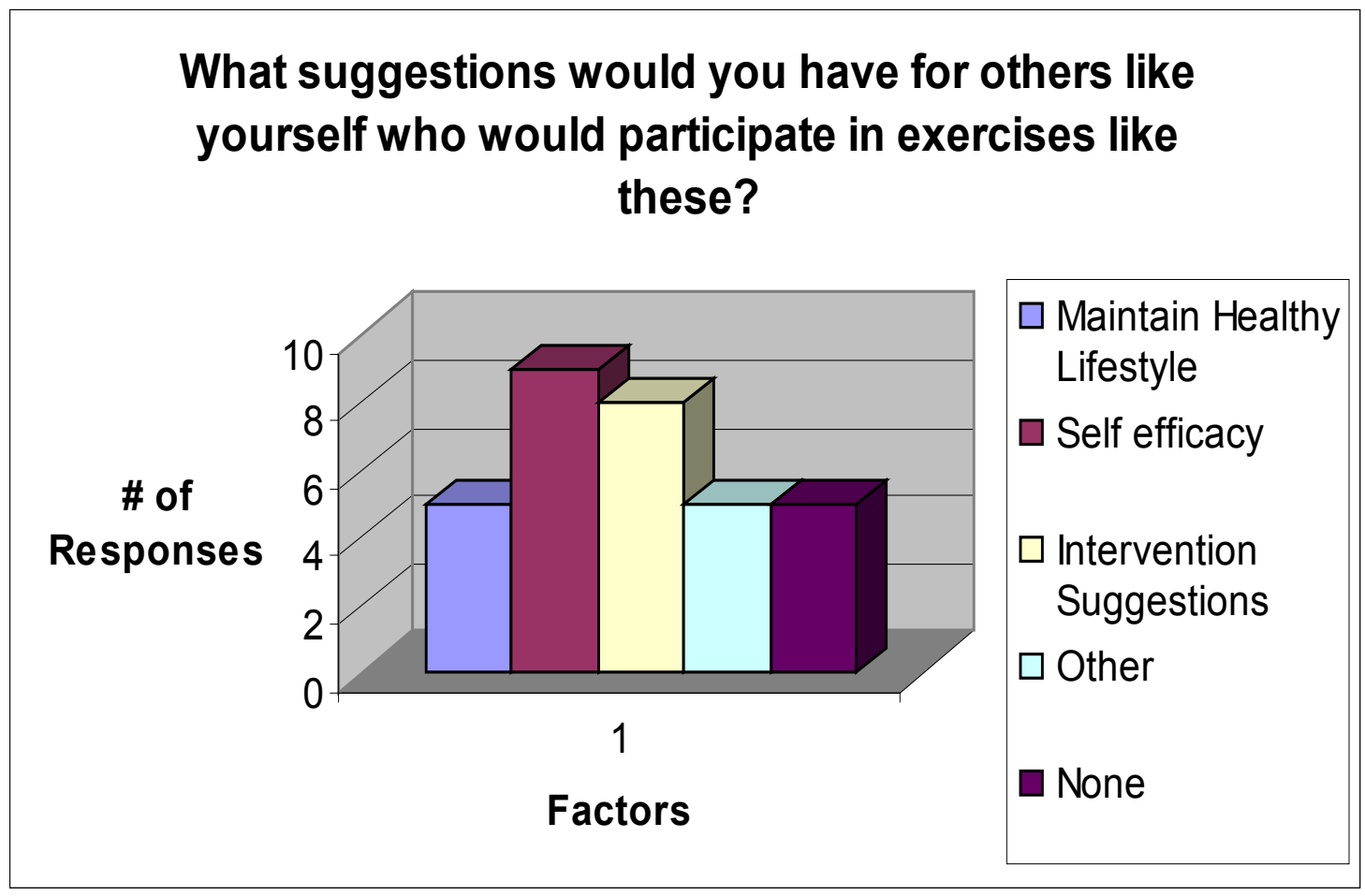

Figure 4. Feasibility Question 4

\section{Results}

Five themes concerning the elements of the intervention were found to be most helpful for continuing to exercise. These themes were: social support for exercise, muscle strength gain, determination, features of the intervention, and helping others. Social support for exercise through consistent interactions with the research team and support of family members were cited most often as the major contributing factor in continuing the exercise program for $28.6 \%$ of the intervention group participants. One woman noted that the exercises would make the person stronger, and renew the desire to regain strength that was lost. Having determination to do the exercises, the determination to regain physical strength, a desire to maintain strength was also a theme noted for this question for $17.9 \%(n=5)$ of women. The features of 
the intervention such as the exercise pictorial guide, exercise diary, convenience of the home-based exercise program, and education concerning the benefits of exercise were denoted as being helpful in maintaining exercise for $35.7 \%(n=10)$ of the intervention group participants. The possibility that findings from the study could be helpful to others was noted as a reason for continuing the exercises (14.3\%).

The second question focused on the challenges that prevented exercising. Four themes related to the challenges for exercise participation were symptoms experienced, fatigue, time constraints, and challenges related to the intervention. Symptoms specific to the side-effects of chemotherapy included neutropenia, infection, loosening of finger and toe nails, nausea, and generalized body aches presented the most challenges to exercise (42.8\%). Fatigue was reported as the second leading challenge to exercise $(25.7 \%)$, reinforcing the unresolved prevalence of chemotherapy-related fatigue. The time commitment required for exercise and finding time to perform the exercises, were also cited as challenges $(22.9 \%)$. One woman cited family responsibilities regarding child care as a challenge to exercising. Challenges related to the intervention included difficulty in performing the exercise with the prescribed weight and not enjoying resistance exercise (8.6\%). Lastly, women noted that symptom management, specifically having adequate post-surgical pain control is needed to engage and continue exercise.

The third question regarding benefits from the exercise program consisted of responses including increased strength and energy, emotional well-being/feeling good. One woman reported being unsure of the benefits of the exercise program. The benefit cited most often $(\mathrm{n}=14 ; 50 \%)$ consisted of increased strength and energy. Emotional well-being/feeling good $(35.7 \% ; n=10)$ was the second leading benefit noted from participating in the exercise program. Additional benefits included motivation and helping others to see the importance of exercise for feeling better through the chemotherapy $(14.3 \% ; n=4)$.

The final question allowed the participants to give suggestions to others like them participating in the exercise intervention. A content analysis revealed 3 themes of maintaining a healthy lifestyle, self-efficacy and intervention suggestions. For maintaining a healthy lifestyle, the themes of needing adequate rest, incorporating the exercise routine into daily life, and the emotional and physical benefits of exercise were noted (15.2\%). The need for self-efficacy were noted by suggestions to try your best, do it for yourself, have patience with your body, and thinking positive thoughts were expressed by the women (27.3\%). Suggestions related to the intervention were to watch television during the exercise, to do the exercises early in the day, to exercise with a partner, and to plan time with the research team for issues related to exercising (24.2\%). One participant thought the intervention would be better implemented if it followed the completion of chemotherapy.

\section{Percentage of Recruitment}

Study recruitment was carried out at two Midwestern Cancer Centers. The combined recruitment rate for both Cancer Centers was $85.4 \%$, with 117 potential study participants approached and 100 women accepting to participate in the study. The high recruitment rate suggests the notion of exercising during chemotherapy to be acceptable to women with breast cancer. Women who elected to participate reported the study held appeal as a mechanism for helping themselves recover from the potential side-effects of chemotherapy treatment, and that the knowledge gained may be helpful to others in the future.

\section{Study Withdrawal}

Of the 47 women randomly assigned to receive the resistance exercise intervention, $13(27.6 \%)$ voluntarily withdrew from of the intervention group. Participants tended to withdraw from the study in the early phase of the intervention. Nine women (19.1\%) withdrew during the first 4 weeks of the study. Two women (4.2\%) withdrew between 4 and 8 weeks and an additional two more (4.3\%) between 8 and 12 weeks. The reasons cited for study withdrawal most often were feeling fatigued, being overwhelmed with the breast cancer diagnosis, and time constraints that interfered with study participation.

\section{Adherence to the Intervention}

Adherence to the resistance exercise intervention was measured using the data from the Exercise Diary reports. As women received the introduction to the exercises and the first diary to complete at the baseline interview, diary data was collected at 4, 8, and 12 weeks. Adherence to the intervention was determined to consist of engaging in the strength training exercises at least two times weekly. Reports of exercise collected via the Exercise Diary declined over the study period. Of the 47 women assigned to the intervention group, the availability of diary data ranges across the study time period from $28(59.5 \%)$ women completing the Exercise Diary at 4 weeks, 25 women $(53.1 \%)$ at 8 weeks and 19 women $(40.4 \%)$ at 12 weeks. In addition to voluntary study withdrawal, reasons for incomplete Exercise Diary data included failure to return the diary to the research staff or record exercise sessions in the diary. In the end, 33 of the original 47 women completed the entire 12 week intervention. At 4 weeks, Exercise Diary data revealed that 27 of the $33(82 \%)$ of women were exercising at least twice weekly. Twenty-three women (69.6\%) reported engaging in resistance exercise sessions 3 times weekly during the first month of the study. Seven women (21.2\%) reported exercising more than three times weekly for at least one of the 4 weeks of the month.

At 8 weeks 24 (72.7\%) of 33 study participants reported exercising at least twice weekly. Fifteen women (45.4\%) reported engaging in resistance exercise sessions 3 times weekly for at least one week during this time period. Six women $(18.1 \%)$ reported exercising more than three times weekly during one week.

At 12 weeks, exercise diary data was available for only 19 
intervention group participants (57.5\%). Eighteen women (94.7\%) reported exercising at least twice weekly for a minimum of one week during the last month. Twelve women (63.2\%) reported exercising 3 times weekly for at least 1 of the 4 weeks. Six women (24\%) exercised more than 3 times a week for at least one week during this last month of the study. Only one (5.3\%) woman reported exercising 3 times weekly for the entire last month of the study. The average adherence rate over the 12 weeks of the study was $84.8 \%$. The data from the Exercise Diaries suggests the majority of women were able to perform the resistance exercises two times weekly, and thus were able to adhere to the resistance exercise intervention.

\section{Discussion}

Study results suggest that a resistance exercise intervention conducted during chemotherapy for breast cancer is both feasible and acceptable. The Participant Evaluation of Feasibility and Acceptability Questionnaire scores indicated that women found the exercise worthwhile, easy to perform, having clear directions and adequate guidance and assistance from the research team regarding the exercise instructions. Only one item of the questionnaire indicated a lack of enjoyment with the prescribed exercise routine, with a mean score of 2.33 . It is possible that the exercises became too routine, lacking the necessary variety to maintain interest. An additional consideration may be that those women who initially reported not engaging in an exercise program of any kind prior to their diagnosis of breast cancer, found the exercise program too much effort at this stressful time.

The remaining 4 additional open ended questions identified the factor most helpful to the women for continuing the exercise program; the challenges that kept women from exercising; the benefits received from the exercise program; and suggestions for others like them participating in a similar exercise program. The study data suggest that social support for exercise to be the most important factor in continuing a resistance exercise program during chemotherapy. Women also reported that interactions with the study's research personnel regarding exercise prescription and implementation, and the support of family and friends, were most helpful. This finding is consistent with other studies of exercise in this population. Considering the willingness of women to consider exercising during breast cancer chemotherapy, future studies should include an exercise 'buddy' and frequent contact with the study team to increase exercise adherence and diary recording rates.

The findings also suggest that study participants can be successfully recruited into a resistance exercise program during chemotherapy for breast cancer. In a trial of combined aerobic and resistance exercise for women receiving adjuvant chemotherapy for breast cancer, Courneya et al (2007) reported a recruitment rate of $33 \%$, while Milne et al (2007) was able to recruit $44.3 \%$ of women who had already completed chemotherapy into a similar exercise program. The $85 \%$ recruitment rate for this study was quite high. However, stronger emphasis needs to be placed on symptom management during the early phases of the study, when most voluntary withdrawal occurred. Symptoms and fatigue related to the effects of chemotherapy were cited as presenting the most challenges to engaging in resistance exercises. Similarly, Courneya et al (2004) reported cancer treatment to have a negative effect on exercise adherence, particularly for those receiving multimodal (i.e. radiation plus chemotherapy). Although women received chemotherapy alone, the treatment was considered fairly intense due to the dose-dense (every 2 week) administration regimen and accompanying side effect profile. Strategies are needed to assist women in overcoming other barriers to exercise. Assisting women to problem-solve issues related to time constraints relative to incorporating exercise into their lives during chemotherapy is needed for future interventions. Also, helping women find exercise routines that they enjoy would increase the likelihood of engaging in regular exercise.

Despite measures to obtain exercise data, study participants failed to compete and return the diary. Maintaining an exercise diary in a consistent manner appeared to become more difficult over time. The recording of exercise sessions 2-3 times weekly may have been perceived as burdensome. Additionally, if the Exercise Diary was not completed in a timely manner, the exercise sessions could be forgotten and resulted in missing data. While women anecdotally reported doing the exercises, intentionally taking time to record data lapsed when exercise patterns seemed to become more firmly established. In future studies, using an exercise check-list, computer entry, or other means of minimizing recording of exercise data may prove more effective. Although data suggests that few women achieved the optimum number of 3 training sessions per week, most women demonstrated consistency with the prescribed exercise guidelines and performed the resistance exercises at least 2 times weekly.

Women engaging in the resistance exercises reported benefits such as physiologic improvements in energy, muscle mass and weight control. To achieve or maintain improvements gained with resistance exercise, increases in both resistance (weight), and number of repetitions is needed. Coaching women to understand the relationship between increased resistance and repetitions and resultant physical improvements may assist in the adoption and maintenance of strength training exercises. There are potential benefits of resistance exercise in improving the adverse neuromuscular side effects of chemotherapy, but clinicians must find a more feasible approach toward exercise maintenance. The importance and benefits of maintaining physical activity and promoting exercise should be discussed with women preparing to undergo chemotherapy for breast cancer. Practitioners should identify lifestyle and time management needs of their patient in order to devise a routine that is consistent with personal schedules. 
Study limitations include subject attrition and missing data. While the study sample could be adequately recruited, addressing chemotherapy-related symptom management with oncology health care providers is needed to retain study participants. The clinical applicability of a strength training program is feasible and acceptable to women undergoing chemotherapy, and can easily be performed in a comfortable setting for the client. In summary, engaging in resistance exercises throughout chemotherapy for breast cancer is both feasible and acceptable, and has the potential to combat the neuromuscular side effects of chemotherapy and manage resulting functional limitations. Weight gain has been particularly problematic for women with breast cancer. Future research is needed to explore how resistance exercises during or after treatment can impact long-term muscle strength and body composition in this population. Aromatase inhibitor therapy often follows chemotherapy for postmenopausal women with breast cancer and is known to induce osteoporosis. Resistance exercise in addition to vitamin $\mathrm{D}$ and calcium supplementation may have a role in increasing bone mineral density and preventing fractures in this population.

\section{Acknowledgements}

This study was funded by a grant from the National Cancer Institute (5R03CA103488-02).

\section{REFERENCES}

[1] Schmitz, K.H., Holtaman, J., Courneya, K.S., Masse, L., Duval, S. \& Kane, R. (2005).Controlled physical activity trials in cancer survivors: A systematic review and meta analysis. Cancer Epidemiology, Biomarkers \& Prevention, 14(7), 1588-1595.

[2] Fairey, A.S., Courneya, K.S., Field, C.J., Bell, G.J., Jones, L.W., \& Mackey, J.R. (2003). Effects of exercise training on fasting insulin, insulin resistance, insulin like growth factors, and insulin-like growth factor binding proteins in postmenopausal breast cancer survivors: A randomized controlled trial. Cancer Epidemiology, Biomarkers \& Prevention, 12: 721-727.

[3] Fairey, A.S., Courneya, K.S., Field, C.J., Bell, G.J., Jones, L.W. \& Mackey, J.R. (2005). Randomized controlled trial of exercise and blood immune function in postmenopausal breast cancer survivors. Journal of Applied Physiology, 98(4), 1534-1540.

[4] Cunningham, B., Morris, G., Cheney, C., Buergel, N., Aker, S., \&Lenssen, P. (1986). The effects of resistive exercise on skeletal muscle in marrow transplant recipients receiving total parenteral nutrition. Journal of Parenteral and Enteral Nutrition, 10(6), 558 - 563.

[5] Winningham, M., \& McVicar, M. (1988). The effect of aerobic exercise on patient reports of nausea. Oncology Nursing Forum, 15(4), 447- 450.
[6] Winningham, M.L., MacVicar, M.G., Bondoc, M., Anderson, J.L., \& Minton, J.P. (1989). Effect of aerobic exercise on body weight and composition in patients with breast cancer an adjuvant chemotherapy. Oncology Nursing Forum, 16 (5), $683-689$.

[7] MacVicar, M., Winningham, M. \& Nickel, J. (1989). Effects of aerobic interval training on cancer patient's functional capacity. Nursing Research, 38(6), 348-351.

[8] Peters, C., Lotzerich, H., Niemeier, B., Schule, K., Uhlenbruck, G. (1994). Influence of a moderate exercise training on natural killer cytotoxicity and personality traits in cancer patients. Anitcancer Research, 14(3A), 1033-1036.

[9] Peters, C., Lotzerich, H., Niemeier, B., Schule, K., Uhlenbruck, G. (1995). Exercise, cancer and the immune response of monocytes. Anticancer Research, 15(1), 175179.

[10] Mock, V., Dow, K.H., Meares, C.J., Grimm, P.M., Dienemann, J.A., Haisfield-Wolfe, M.E., Quitasol, W., Mitchell, S., Chakravarthy, A., \& Gage, I. (1997). Effects of exercise on fatigue, physical functioning, and emotional distress during radiation therapy for breast cancer. Oncology Nursing Forum, 24(6), 991-1000.

[11] Segar, M.L., Katch, V.L., Roth, R.S., Garcia, A., Portner, T., Glickman, S., Haslanger, S., \& Wilkens, E. (1998). The effect of aerobic exercise of self esteem and depressive and anxiety symptoms among breast cancer survivors. Oncology Nursing Forum, 25(1), 107-113.

[12] Schwartz, A. L. (1999). Fatigue mediates the effects of exercise on quality of life. Quality of Life Research, 8: 529-538.

[13] Schwartz, A.L., Motomi, M., Gao, R., Nail, L.M., \& King, M.E. (2001). Exercise reduces daily fatigue in women with breast cancer receiving chemotherapy. Medicine \& Science in Sports \& Exercise, 33(5), 718-723.

[14] Segal, R., Evans, W., Johnson, D., Smith, J., Colleta, S., Gayton, J., Woodard, S., Wells, G. \& Reid, R. (2001). Structured exercise improves physical functioning in women with stages I and II breast cancer: Results of a randomized controlled trial. Journal of Clinical Oncology, 19(3), 657-665.

[15] Mock, V., Frangakis, C., Davidson, N., Ropka, M., Pickett, M., Poniatowski, B., Stewart, K.J., Cameron, L., Zwacki, K., Podewils, L.J., Cohen, G. \& McCorkle, R. (2005). Exercise manages fatigue during breast cancer treatment: A randomized controlled trial. Psycho-oncology, 14, 464-477.

[16] Schmitz, K.H., Ahmed, R.L., Hannan, P.J., \& Yee, D. (2005). Safety and efficacy of weight training in recent breast cancer survivors to alter body composition, insulin, and insulin-like growth factor axis proteins. Cancer, Epidemiology, Biomarkers \& Prevention, 14(7), 1672-1680.

[17] Ohira, T., Schmitz, K. H., Ahmed, R.L. \& Yee, D. (2006). Effects of weight training on quality of life in recent breast cancer survivors. Cancer, 106(9), 2076-83.

[18] Waltman, N.L., Twiss, J.J., Ott, C.D., Lindsey, A.M., Moore, T.E. \& Berg, K. (2003). Testing an intervention for preventing osteoporosis in postmenopausal breast cancersurvivors. Journal of Nursing Scholarship, 35(4):333-8.

[19] Ahmed, R.L., Thomas, W., Yee, D. \& Schmitz, K.H. (2006). 
Randomized controlled trial of weight training and lymphedema in breast cancer survivors. Journal of Clinical Oncology, 24(18):2765-72.

[20] Zima, T., Tesar, V., Richardson, P.J., Mantle, D. \& Preedy, V. R. (2001). Effects Doxorubicin (Adriamycin) and [(+)-1,2-bis (3,5-dioxopiperazinyl-1-yl) propane (ICRF-187) on skeletal muscle protease activities. Toxicology and Applied Pharmacology, 171(3), 135-140.

[21] Sandler, L. \& Vernikos, J. (1986). Inactivity: Physiological effects. Orlando, Florida: Academic Press.

[22] Bloomfield, S.A. (1997). Changes in musculoskeletal structure and function with prolonged bed rest. Medical Science in Sports and Exercise, 29, 197-206.

[23] Ojala, B., Page, L., Moore, M. \& Thompson, L. (2001). Effects of inactivity on glycolytic capacity of single skeletal muscle fibers in adult and aged rats. Biological Research for Nursing, 3 (2), 88-95.

[24] Piper, B.F., Dibble, S.L., Dodd, M.J., Weiss, M.C., Slaughter,R.E., \& Paul, S.M. (1998). The revised Piper Fatigue Scale: psychometric evaluation in women with breast cancer. Oncology Nursing Forum, 25(4), 677-84.

[25] Ware, J. (2000). SF-36 Health Survey update. Spine, 25, $3130-3339$.

[26] Milne, H.M., Wallman, K.E., Gordon, S., \& Courneya, K.S. (2008). Effects of a combined aerobic and resistance exercise program in breast cancer survivors: a randomized controlled trial. Breast Cancer Research and Treatment, 108(2), 279-88.

[27] Courneya, K.S., Segal, R.J, Mackey, J.R., Gelmon, K., Reid, R.D., Friedenrich, C.M., Ladha, A.B., Proulx, C., Vallance, J.K.H., Lane, K., Yasui, Y., \& McKenzie, D. (2007). Effects of aerobic and resistance exercise in breast cancer patients receiving adjuvant chemotherapy: A multicenter randomized controlled trial. Journal of Clinical Oncology, 25(28), 4396-4404.

[28] Berger, A.M., VonEssen, S., Kuhn, B.R., Piper, B.F., Farr, L., Agrawal, S., Lynch, J.C., \& Higginbotham, P. (2003). Adherence, sleep, and fatigue outcomes after adjuvant breast cancer chemotherapy: Results of a feasibility intervention study. Oncology Nursing Forum, 30(3), 513-22.

[29] Berger, A.M., VonEssen, S., Kuhn, B., Piper, B.F., Farr, L., Agrawal, S., Lynch, J.C., \& Higginbotham, P. (2002). Feasibility of a sleep intervention during adjuvant breast cancer chemotherapy. Oncology Nursing Forum, 29(10), 1431-41.

[30] Von Gruenigen, V.E., Courneya, K.S., Gibbons, H.E., Kavanagh, M.B., Waggoner, S.E., \& Lerner, E. (2008). Feasibility and effectiveness of a lifestyle intervention program in obese endometrial cancer patients: A randomized trial. Gynecologic Oncology, 109(1), 19-26.

[31] Courneya, K.S., Friedenreich, C.M., Quinney, A., Fields, A.L.A, Jones, L.W., \& Fairey, A.S. (2004). Predictors of adherence and contamination in a randomized trial of exercise in colorectal cancer survivors. Psycho-Oncology, 13(12), 857-66.

[32] Courneya, K.S., Friedenreich, C.M., Sela, R.A., Quinney, H.A., \& Rhodes, R.E. (2002). Correlates of adherence and contamination in a randomized controlled trial of exercise in cancer survivors: An application of the theory of planned behavior and the five factor model of personality. Annals of Behavioral Medicine, 24(4), 257-68.

[33] Courneya, K.S., Blanchard, C.M., \& Laing, D.M. (2001). Exercise adherence in breast cancer survivors training for a dragon boat race competition: A preliminary investigation. Psycho-Oncology, 10(5), 444-52. 